\title{
Identification of miRNA and genes involving in osteosarcoma by comprehensive analysis of microRNA and copy number variation data
}

\author{
TAO LUO ${ }^{1}$, XIANGLI YI ${ }^{2}$ and WEI SI $^{2}$ \\ ${ }^{1}$ Department of Blood Transfusion, Tianjin Hospital, Tianjin 300211; ${ }^{2}$ Department of Intensive Care \\ Unit, Tianjin Medical University Cancer Institute and Hospital, Tianjin 300060, P.R. China
}

Received December 24, 2015; Accepted July 5, 2017

DOI: $10.3892 / \mathrm{ol} .2017 .6845$

\begin{abstract}
The aim of the present study was to understand the molecular mechanisms of osteosarcoma by comprehensive analysis of microRNA (miRNA/miR) and copy number variation (CNV) microarray data. Microarray data (GSE65071 and GSE33153) were downloaded from the Gene Expression Omnibus. In GSE65071, differentially expressed miRNAs between the osteosarcoma and control groups were calculated by the Limma package. Target genes of differentially expressed miRNAs were identified by the starBase database. For GSE33153, PennCNV software was used to perform the copy number variation (CNV) analysis. Overlapping of the genes in CNV regions and the target genes of differentially expressed miRNAs were used to construct miRNA-gene regulatory network using the starBase database. A total of 149 differentially expressed miRNAs, including 13 downregulated and 136 upregulated, were identified. In the GSE33153 dataset, 987 CNV regions involving in 3,635 genes were identified. In total, 761 overlapping genes in 987 CNV regions and in the genes in 7,313 miRNA-gene pairs were obtained. miRNAs (hsa-miR-27a-3p, hsa-miR-124-3p, hsa-miR-9-5p, hsa-miR-182-5p, hsa-miR-26a-5p) and the genes [Fibroblast growth factor receptor substrate 2 (FRS2), coronin 1C (CORO1C), forkhead box P1 (FOXP1), cytoplasmic polyadenylation element binding protein 4 (CPEB4) and glucocorticoid induced 1 (GLCCII)] with the highest degrees of association with osteosarcoma development were identified. Hsa-miR-27a-3p, hsa-miR-9-5p, hsa-miR-182-5p, FRS2, CORO1C, FOXP1 and CPEB4 may be involved in osteosarcoma pathogenesis, and development.
\end{abstract}

Correspondence to: Mr. Tao Luo, Department of Blood Transfusion, Tianjin Hospital, 406 Jie-Fang-Nan Road, Tianjin 300211, P.R. China E-mail: weisisummer@163.com

Key words: osteosarcoma, microRNA, copy number variation, microRNA-gene regulatory network

\section{Introduction}

Osteosarcoma is the most common type of primary bone tumor in adolescents and young adults $(1,2)$, and is characterized by an abundance of genomic aberrations (3). According to previous studies, the 5-year survival rate of osteosarcoma is $60-70 \%$, and the prognosis has not significantly improved over the last 30 years (4). Despite improvements in osteosarcoma treatment, the molecular mechanism underlying osteosarcoma development remains unclear. Therefore, it is important to explore the molecular mechanism of osteosarcoma development to additionally improve osteosarcoma treatment. However, the mechanisms of osteosarcoma development are complex, and numerous factors, including genes associated with osteosarcoma (3), copy number variations (CNVs) across the whole genome (5) and miRNAs (6), may contribute to the development of osteosarcoma. In particular, CNV may contribute to the development of various types of cancer, including osteosarcoma and B-cell lymphoma $(7,8)$. CNV has been considered as a marker for cancer predisposition (9). CNV may contribute to the pathogenesis of osteosarcoma (10) and the disease risk for diffuse large B-cell lymphoma (11).

Furthermore, a number of previous studies revealed that microRNAs (miRNAs/miRs) are associated with the development of osteosarcoma $(12,13)$, and may be used as the molecular targets for osteosarcoma, including miR-143, miR-382 and miR-214. Downregulation of miR-143 is associated with the lung metastasis of osteosarcoma via the upregulated expression of matrix metalloproteinase 13 (14). Overexpression of miR-382 may suppress the metastasis of osteosarcoma (15). Upregulated expression of miR-214 may contribute to the pathogenesis of osteosarcoma and may be associated with adverse prognosis (16). In addition, miRNAs, including miR-143 (17), miR-27a (18), miR-223 (19), miR-191 (20), miR-133a (21) and miR-26a (22), may also be associated with the development of osteosarcoma. These results indicated that miRNAs may serve an important role in the pathogenesis of osteosarcoma.

Previously, miRNAs expression profiles $(23,24)$ and SNP microarray (1) were used to identify the genes, miRNAs, and single nucleotide polymorphisms (SNPs) associated with the development of osteosarcoma. In certain studies, specific genes 
associated with osteosarcoma development were identified by the integrative analysis of copy number and gene expression data (25). Based on the aforementioned, comprehensive analysis of microRNA (GSE65071) and CNV (GSE33153) microarray data were performed in the present study to identify the miRNAs, and genes involved in the pathogenesis of osteosarcoma. In addition, a miRNA-gene regulatory network of the overlapping genes of the genes in CNV regions and target genes of differentially expressed miRNAs was constructed. These miRNAs and genes associated with osteosarcoma development were identified in order to additionally explore the mechanism of osteosarcoma, and may be used as candidate target miRNAs and genes for gene therapy.

\section{Materials and methods}

Microarray data. Microarray data (GSE65071 and GSE33153) were downloaded from Gene Expression Omnibus (GEO, http://www.ncbi.nlm.nih.gov/geo/). For GSE65071, there were 20 osteosarcoma samples and 10 healthy control samples, and the platform was GPL19631Exiqon human V3 microRNA PCR panel I+II. A total of 32 osteosarcoma samples were available on GSE33153, of which the platform was GPL6801 [GenomeWideSNP_6] Affymetrix Genome-Wide Human SNP 6.0 Array.

Data preprocessing and analysis of copy number variation. GSE65071 was preprocessed using preprocessCore package v1.38.1 (http://www.bioconductor.org/packages/release/bioc/html/preprocessCore.html) in $\mathrm{R}$ language. Then, the differentially expressed miRNAs between the osteosarcoma and control groups were calculated using the Limma package v3.32.2 (http://www.bioconductor.org/packages/release/bioc/html/limma.html), and the adjusted P-value $<0.05$ and $\log$ (fold change) $\mid>2$ were chosen as the cut-off criterion. Then, two-way clustering analysis was performed using the gplots package v3.0.1 (https://cran.r-project.org/web/packages/gplots/index.html).

For GSE33153, the $\log$ R Ratio (LRR) and B allele frequency (BAF) were extracted using the affy 2 sv package v1.0.12 (https://bitbucket.org/brge/affy $2 \mathrm{sv}$ ), and then CNV calling was performed using PennCNV v2014 (07) (http://penncnv.openbioinformatics.org/). CNV identified in $\geq 4$ samples was considered as $\mathrm{CNV}$ regions, which may be involved in the development of osteosarcoma.

Identification of target genes of miRNAs. The regulatory associations between long non-coding RNA, miRNA, competing endogenous RNA, mRNA and RNA binding proteins may be identified using the starBase database (http://starbase.sysu.edu .cn/), which included the clinical data and expression profiles of 14 types of cancer. The target genes of differentially expressed miRNAs were identified using the starBase database. The cut-off criterion of miRNA-gene pairs, which were used to screen the target genes, was listed as follows: i) miRNA-gene pairs were confirmed by $\geq 2$ experiments; and ii) the change in the trend of miRNA and target gene expression values was the opposite.

Comprehensive analysis of miRNAs and $C N V$ regions. Genes in the CNV regions were screened using scan_region.pl of
Table I. Top ten differentially expressed miRNAs.

\begin{tabular}{lccc}
\hline miRNAs & $\operatorname{logFC}$ & P-value & $\begin{array}{c}\text { Adjusted } \\
\text { P-value }\end{array}$ \\
\hline hsa-miR-624-5p & 4.998135150 & $2.08 \times 10^{-30}$ & $7.41 \times 10^{-28}$ \\
hsa-miR-505-5p & 3.631886325 & $2.96 \times 10^{-28}$ & $5.27 \times 10^{-26}$ \\
hsa-let-7f-2-3p & 3.613251767 & $4.96 \times 10^{-28}$ & $5.89 \times 10^{-26}$ \\
hsa-miR-877-3p & 4.267538662 & $4.38 \times 10^{-27}$ & $3.89 \times 10^{-25}$ \\
hsa-miR-183-5p & 4.469024403 & $1.94 \times 10^{-26}$ & $1.26 \times 10^{-24}$ \\
hsa-miR-342-5p & 5.006618179 & $2.12 \times 10^{-26}$ & $1.26 \times 10^{-24}$ \\
hsa-let-7f-1-3p & 3.182939079 & $8.79 \times 10^{-26}$ & $4.47 \times 10^{-24}$ \\
hsa-miR-671-5p & 5.476974410 & $1.58 \times 10^{-25}$ & $7.02 \times 10^{-24}$ \\
hsa-miR-95 & 5.163185811 & $2.96 \times 10^{-25}$ & $1.13 \times 10^{-23}$ \\
hsa-miR-499a-5p & 5.429803038 & $3.18 \times 10^{-25}$ & $1.13 \times 10^{-23}$ \\
\hline
\end{tabular}

miRNAs/miR, microRNA; hsa, Homo sapiens.

PennCNV. The overlap between genes contained within the CNV regions and targets of the differentially expressed miRNAs were obtained. miRNA-gene pairs that overlapped were obtained based on the starBase database, and a miRNA-gene regulatory network was constructed.

\section{Results}

Analysis of differentially expressed miRNA and CNV regions. A total of 149 differentially expressed miRNAs between the osteosarcoma group and the control group were identified, including 13 downregulated, and 136 upregulated differentially expressed miRNAs (Table I). The clustering plot indicated that the differentially expressed miRNAs evidently separated the osteosarcoma samples from the control samples (Fig. 1).

For GSE33153, 987 CNV regions were identified. The distribution of the $987 \mathrm{CNV}$ regions among chromosomes is presented in Fig. 2. In Fig. 2, the red and blue column represents the deletions, and overlap of copy number, respectively. The longer the column, the higher the number of samples that exhibited deletions or overlapping in these CNV regions.

Analysis of target genes of differentially expressed miRNAs. In total, 7,313 miRNA-gene pairs were screened for 149 differentially expressed miRNAs, and 5 differentially expressed miRNAs (hsa-miR-200b-3p, hsa-miR-200a-3p, hsa-miR-429, hsa-miR-34a-5p, and hsa-miR-9-5p) with corresponding target genes were identified, as summarized in Table II.

Comprehensive analysis of differentially expressed miRNA and CNV regions. A total of 761/3,635 genes in $987 \mathrm{CNV}$ regions and the genes of 7,313 miRNA-gene pairs were identified overlap. The miRNAs with highest degrees of overlap (hsa-miR-27a-3p degree, 112; hsa-miR-124-3p degree, 102; hsa-miR-9-5p degree, 90; hsa-miR-182-5p degree, 79; hsa-miR-26a-5p degree, 79) and genes (FRS2 degree, 14; CORO1C degree, 12; FOXP1 degree, 11; CPEB4 degree, 11; GLCCI1 degree, 10) are presented in Table III. 

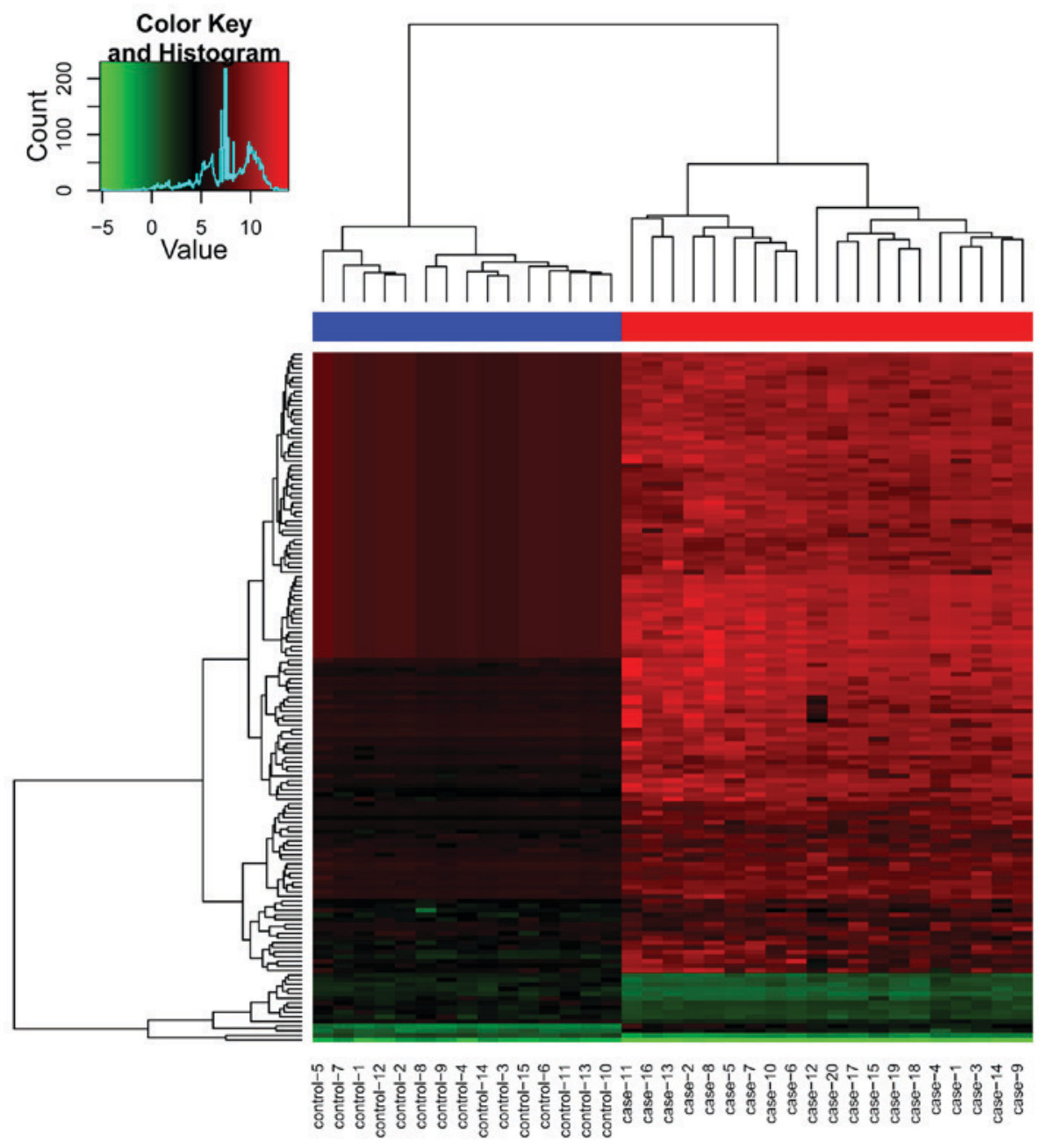

Figure 1. Clustering plot of the differentially expressed microRNAs when comparing the osteosarcoma group and the control group. Green and red colors represent the low, and high expression values, respectively.
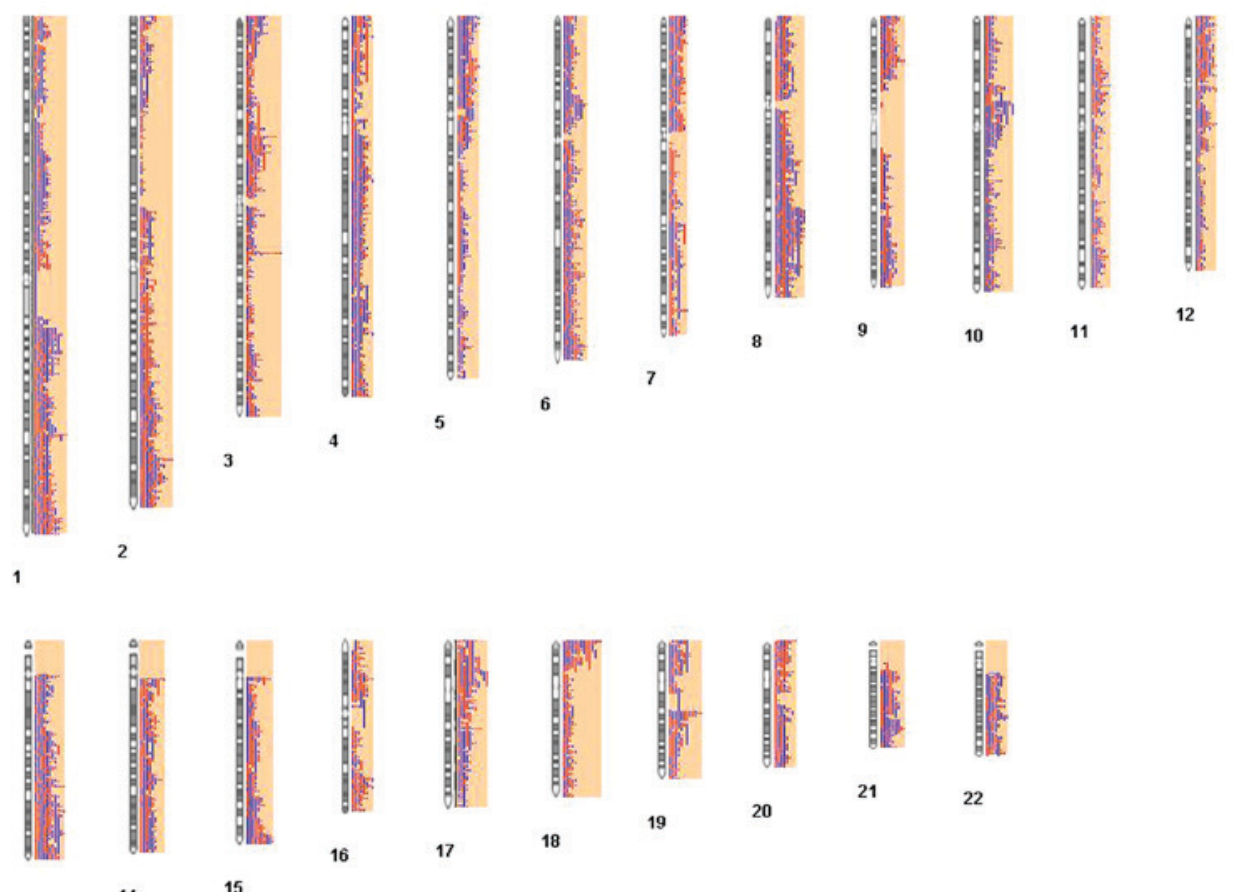

13

Figure 2. Distribution of 987 CNV regions among chromosomes. Red and blue column represented the deletions, and overlap of copy numbers, respectively. The longer column, the higher the number of samples that exhibited deletions or overlapping in these CNV regions. CNV, copy number variation. 
Table II. miRNA-target gene pairs.

\begin{tabular}{lcl}
\hline miRNAs & Counts & \multicolumn{1}{c}{ Target genes $^{\mathrm{a}}$} \\
\hline hsa-miR-200b-3p & 282 & ICK, NPTX1, DPY19L1, RECK, TRIO, DDX3Y, GALNT2, R3HDM2, PPM1B, TIMP2 \\
hsa-miR-200a-3p & 159 & GIGYF1, GIGYF1, MTF2, CASC4, ULK2, MBNL3, KPNA4, ZEB2, ZEB2, TET3 \\
hsa-miR-429 & 235 & SEC24A, TRIM52, DPY19L1, PAPOLA, GALNT2, KIAA1432, MEX3C, PLCG1, \\
& 92 & LRIG1, DCBLD2 \\
hsa-miR-34a-5p & TNRC18, SIDT2, ARPP19, NAA50, RCAN1, DNAJB1, XPO5, HCN3, SIRT1, MBD6 \\
hsa-miR-9-5p & 144 & ULK2, SMARCE1, ZC3H10, VAT1, BCAT2, DICER1, ZC3H12A, GTPBP3, \\
& & SH2B3, ITM2B
\end{tabular}

${ }^{a}$ The genes listed do not cover the exhaustive group of target genes for each miRNA. miRNAs/miR, microRNA; hsa, Homo sapiens. Counts represent the number of target genes which were contained within the CNV regions.

\section{Discussion}

Osteosarcoma is characterized by an abundance of genomic aberrations (3). In the present study, a comprehensive analysis of microRNA data and CNV microarray data was performed to identify miRNAs, and genes associated with the pathogenesis of osteosarcoma. A total of 149 differentially expressed miRNAs, including 13 down- and 136 upregulated, in the GEO GSE65071 dataset were identified. For the GEO GSE33153 dataset, $987 \mathrm{CNV}$ regions were identified. In addition, a miRNA-gene regulatory network of 761 overlapping genes out of 3,635 genes in $987 \mathrm{CNV}$ regions and the genes in 7,313 miRNA-gene pairs was constructed. Concurrently, miRNAs (hsa-miR-27a-3p, hsa-miR-124-3p, hsa-miR-9-5p, hsa-miR-182-5p, hsa-miR-26a-5p) and genes [fibroblast growth factor receptor substrate 2 (FRS2), coronin 1C (CORO1C), forkhead Box P1 (FOXP1), cytoplasmic polyadenylation element binding protein 4 (CPEB4) and glucocorticoid induced 1 (GLCCII)] with the highest degree of overlap in the miRNA-gene regulatory network were identified. Higher degrees represent an increased level of association to the development of osteosarcoma. Therefore, hsa-miR-27a-3p, hsa-miR-124-3p, hsa-miR-9-5p, hsa-miR-182-5p, hsa-miR-26a-5p, FRS2, COROIC, FOXP1, $C P E B 4$ and GLCCI1 may contribute to the pathogenesis of osteosarcoma.

The expression of hsa-miR-27a-3p serves an important role in the development of a number of types of cancer, including breast and pancreatic cancer. The expression of hsa-miR-27a-3p was positively correlated with a hypoxia gene signature in breast cancer (26). The inhibition of hsa-miR-27a-3p expression may exhibit potentially antiproliferative effects in pancreatic cancer (27). In addition, hsa-miR-27a-3p was considered as a candidate biomarker for Alzheimer's disease (28). However, the correlation between hsa-miR-27a-3p and osteosarcoma has not been widely investigated. In the present study, hsa-miR-27a-3p was identified as a hub gene with a high degree of association in miRNA-gene regulatory network. In combination with previous studies, the present study hypothesized that hsa-miR-27a-3p may serve a role in the development of osteosarcoma.

Hsa-miR-9-5p, hsa-miR-155-5p and hsa-miR-203 are potent prognostic factors for acute myeloid leukemia (29). In addition, a
3-miRNA scoring system (hsa-miR-9-5p, hsa-miR-155-5p and hsa-miR-203) was used for the prognostication of the patients with de novo acute myeloid leukemia (30). Hsa-miR-182-5p has been considered as a marker for distinguishing between human ovarian cancer tissues and normal tissues (31). The knockdown of miR-182-5p significantly decreased the growth of prostate tumor, and $F O X F 2$, reversion inducing cysteine rich protein with kazal motifs (RECK) and metastasis suppressor 1 were identified as potential target genes of miR-182-5p (32). Previous studies have also revealed that mothers against decapentaplegic homolog 4 (Smad4) and RECK were the potential target genes of miR-182-5p, and that miR-182-5p served a key role in bladder cancer by knocking down the expression of Smad4 and RECK (33). In the present study, hsa-miR-9-5p and hsa-miR-182-5p were identified as the hub genes with high degrees of association in the miRNA-gene regulatory network, implying that hsa-miR-9-5p and hsa-miR-182-5p may serve a key role in the pathogenesis of osteosarcoma.

In the miRNA-gene regulatory network, FRS2, COROIC, FOXP1, CPEB 4 and GLCCI1 with high degrees of association were identified, implying that these genes may be associated with the regulatory mechanism of osteosarcoma. FRS2 is considered to be a gene that is associated with numerous types of cancer, including ovarian cancer, liposarcoma and prostate cancer (34-36). FRS2 serves as an amplified oncogene that induces the downstream activation of the Ras-mitogen-activated protein kinase pathway in high-grade serous ovarian cancer (36). In addition, FRS2 serves an essential role in fibroblast growth factor receptor (FGFR) signaling, and activated FGFR/FRS2 signaling may lead to the development of high-grade liposarcoma (34). Concomitantly, a previous study also identified that the functional overlap of $F R S 2$ and $F R S 3$ may mediate mitogenic FGF signaling in prostate cancer (37). From these data, the present study hypothesized that $F R S 2$ was involved in the regulatory mechanism of osteosarcoma.

COROIC is a target gene of miR-206 that has been demonstrated to inhibit cell migration in triple-negative breast cancer (38). COROIC is also considered as a target gene of miR-1/133a following genome-wide gene expression and luciferase reporter assay analyses (39). Furthermore miR-1 and miR-133a inhibited the proliferation, migration, and invasion of lung-squamous cell carcinoma cells (39). In addition, 
Table III. miRNAs and genes in the miRNA-gene regulatory network.

A, miRNAs in the miRNA-gene regulatory network

\begin{tabular}{|c|c|c|c|}
\hline Marker & Degrees & Counts & Target genes ${ }^{\mathrm{a}}$ \\
\hline hsa-miR-27a-3p & 112 & 51 & $\begin{array}{l}\text { TNRC18, PPARA, ACLY, DNAJB9, NR2F2, HMGCS1, } \\
\text { SGMS1, CKAP4, RPN2, CALM }\end{array}$ \\
\hline hsa-miR-124-3p & 102 & 42 & $\begin{array}{l}\text { SGMS1, DRAM1, SUCLG2, FRS2, NUDCD2, FAR1, } \\
\text { PIP4K2C, SERTAD3, SH3PXD2A, KLHL24 }\end{array}$ \\
\hline hsa-miR-9-5p & 90 & 39 & $\begin{array}{l}\text { FREM2, SMARCE1, KLHDC10, DYRK1B, SLC39A14, } \\
\text { FURIN, BAHD1, MAPKAPK2, AP2M1, BCL6 }\end{array}$ \\
\hline hsa-miR-182-5p & 79 & 38 & $\begin{array}{l}\text { RTN4, STARD13, FRS2, KIAA1217, CNOT6, EXOC4, } \\
\text { SNAP23, KCMF1, QSER1, SYPL1, BDNF }\end{array}$ \\
\hline hsa-miR-26a-5p & 79 & 29 & $\begin{array}{l}\text { SRP19, MARK1, SEMA6D, ACSL3, EIF4G2, LSM12, } \\
\text { MAPK6, CCNJL, MFHAS1, COX5A }\end{array}$ \\
\hline hsa-miR-429 & 79 & 32 & $\begin{array}{l}\text { SUZ12, CRKL, TOB1, FXR1, LIN7B, EVI5, GLCCI1, FRS2, } \\
\text { ARID4B, SSH2 }\end{array}$ \\
\hline hsa-miR-141-3p & 71 & 30 & $\begin{array}{l}\text { RHEB, LENG8, ATP2A2, BAHD1, GLCCI1, ATP1B1, } \\
\text { PTPRG, MBTD1, GRIN2D, STAT5B }\end{array}$ \\
\hline hsa-miR-96-5p & 70 & 33 & $\begin{array}{l}\text { ARPP19, CELSR1, KIAA1217, DOCK1, CAPNS1, CCNG1, } \\
\text { PROK2, APPL1, PGAP1, SHC1 }\end{array}$ \\
\hline hsa-miR-34a-5p & 63 & 30 & $\begin{array}{l}\text { HCN3, HECW2, STC1, MAP2K1, APH1A, NUMBL, } \\
\text { NFE2L1, GREM2, ARID4B, LGR4 }\end{array}$ \\
\hline hsa-miR-200a-3p & 63 & 27 & $\begin{array}{l}\text { RHEB, NCKAP5, B3GNT5, LENG8, CALU, ATP2A2, } \\
\text { BAHD1, NRP1, SPAG9, GLCCI1 }\end{array}$ \\
\hline
\end{tabular}

$\mathrm{B}$, Genes in the miRNA-gene regulatory network

\begin{tabular}{|c|c|c|c|}
\hline Marker & Degrees & Counts & Target genes \\
\hline FRS2 & 14 & - & - \\
\hline CORO1C & 12 & - & - \\
\hline FOXP1 & 11 & - & - \\
\hline CPEB4 & 11 & - & - \\
\hline GLCCI1 & 10 & - & - \\
\hline CELF1 & 10 & - & - \\
\hline MET & 10 & - & - \\
\hline ZFHX4 & 9 & - & - \\
\hline DOCK4 & 8 & - & - \\
\hline MYH10 & 8 & - & - \\
\hline
\end{tabular}

${ }^{a}$ The genes listed do not cover the exhaustive group of target genes for each miRNA. miRNAs/miR, microRNA; hsa, Homo sapiens. Counts represent the number of target genes which were contained within the $\mathrm{CNV}$ regions.

miR-1 and miR-133b mediated cell proliferation, and cell cycle progression by regulating the expression of hepatocyte growth factor receptor protein in human osteosarcoma (40). miR-133b and miR-206 expression were identified to be significantly decreased and may be used as potential prognostic markers for patients with osteosarcoma (41). Decreased miR-206 expression is associated with the development of osteosarcoma, and the transfection of miR-206 mimics promoted cell apoptosis, and inhibited cell invasion and migration (42). COROIC may serve as the target gene of miR-1, miR-133b and miR-206, which have all been associated with osteosarcoma development. Furthermore, COROIC was identified as a node gene with a high degree of association in the miRNA-gene regulatory network. However, COROIC was not identified as a target gene of the miRNAs in the present study. Together, the results indicate that $C O R O 1 C$ may serve a role in osteosarcoma pathogenesis and development by regulating miRNAs.

FOXP1 expression is associated with the development of osteosarcoma $(43,44)$. CPEB4 serves a role in metastatic cancer and cancer progression $(45,46)$; however, whether $C P E B 4$ is involved in the pathogenesis of osteosarcoma remains unclear. In the present study, FOXPI and $C P E B 4$ were also identified as the node genes with high degrees of association in the miRNA-gene regulatory network. Based on the results, it was 
hypothesized that $F O X P 1$ and $C P E B 4$ may contribute to osteosarcoma progression, and development. However, these results require additional confirmation.

In the present study, hsa-miR-27a-3p, hsa-miR-9-5p, hsa-miR-182-5p, FRS2, COROIC, FOXP1 and CPEB4 were identified as node genes with high degrees of association in the miRNA-gene regulatory network, and may serve a role in the pathogenesis and development of osteosarcoma.

\section{References}

1. Savage SA, Mirabello L, Wang Z, Gastier-Foster JM, Gorlick R, Khanna C, Flanagan AM, Tirabosco R, Andrulis IL, Wunder JS, et al: Genome-wide association study identifies two susceptibility loci for osteosarcoma. Nat Genet 45: 799-803, 2013.

2. Kansara M and Thomas DM: Molecular pathogenesis of osteosarcoma. DNA Cell Biol 26: 1-18, 2007.

3. Both J, Krijgsman O, Bras J, Schaap GR, Baas F, Ylstra B and Hulsebos TJ: Focal chromosomal copy number aberrations identify CMTM8 and GPR177 as new candidate driver genes in osteosarcoma. PLoS One 9: e115835, 2014.

4. Broadhead ML, Clark JC, Myers DE, Dass CR and Choong PF: The molecular pathogenesis of osteosarcoma: A review. Sarcoma 2011: 959248, 2011.

5. Zong C, Lu S, Chapman AR and Xie XS: Genome-wide detection of single-nucleotide and copy-number variations of a single human cell. Science 338: 1622-1626, 2012.

6. Jin J, Cai L, Liu ZM and Zhou XS: miRNA-218 inhibits osteosarcoma cell migration and invasion by down-regulating of TIAM1, MMP2 and MMP9. Asian Pac J Cancer Prev 14: 3681-3684, 2013.

7. Zhang H, Wang Z, Bao L, et al: The significance of copy number variation in multiple osteosarcoma's malignance grade, drug resistance and classification. Mar Sci Bull 24: 41-46, 2015.

8. Cui W, Cai Y, Wang W, Liu Z, Wei P, Bi R, Chen W, Sun M and Zhou X: Frequent copy number variations of PI3K/AKT pathway and aberrant protein expressions of PI3K subunits are associated with inferior survival in diffuse large B cell lymphoma. J Transl Med 12: 10, 2014

9. Silva AG, Krepischi AC, Pearson PL, Hainaut P, Rosenberg C and Achatz MI: The profile and contribution of rare germline copy number variants to cancer risk in Li-Fraumeni patients negative for TP53 mutations. Orphanet J Rare Dis 9: 63, 2014.

10. Gokgoz N, Wunder JS and Andrulis IL: Abstract 5075 Genome-wide analysis of DNA copy number variations in osteosarcoma. Can Res 72: 5075, 2012.

11. Conde L, Riby J, Zhang J, Bracci PM and Skibola CF: Copy number variation analysis on a non-Hodgkin lymphoma case-control study identifies an 11q25 duplication associated with diffuse large B-cell lymphoma. PLoS One 9: e105382, 2014.

12. Shimada M: MicroRNA-mediated regulation of apoptosis in osteosarcoma. J Carcinog Mutagen, 2013. doi: 10.4172/2157-2518 S6-001.

13. Jones KB, Salah Z, Del Mare S, Galasso M, Gaudio E, Nuovo GJ, Lovat F, LeBlanc K, Palatini J, Randall RL, et al: miRNA signatures associate with pathogenesis and progression of osteosarcoma. Cancer Res 72: 1865-1877, 2012.

14. Osaki M, Takeshita F, Sugimoto Y, Kosaka N, Yamamoto Y, Yoshioka Y, Kobayashi E, Yamada T, Kawai A, Inoue T, et al: MicroRNA-143 regulates human osteosarcoma metastasis by regulating matrix metalloprotease-13 expression. Mol Ther 19: 1123-1130, 2011.

15. Xu M, Jin H, Xu CX, Sun B, Song ZG, Bi WZ and Wang Y: miR-382 inhibits osteosarcoma metastasis and relapse by targeting Y box-binding protein 1. Mol Ther 23: 89-98, 2015.

16. Wang Z, Cai H, Lin L, Tang M and Cai H: Upregulated expression of microRNA-214 is linked to tumor progression and adverse prognosis in pediatric osteosarcoma. Pediatr Blood Cancer 61: 206-210, 2014

17. Shimbo K, Miyaki S, Ishitobi H, Kato Y, Kubo T, Shimose S and Ochi M: Exosome-formed synthetic microRNA-143 is transferred to osteosarcoma cells and inhibits their migration. Biochem Biophys Res Commun 445: 381-387, 2014.
18. Salah Z, Arafeh R, Maximov V, Galasso M, Khawaled S, Abou-Sharieha S, Volinia S, Jones KB, Croce CM and Aqeilan RI: miR-27a and miR-27a* contribute to metastatic properties of osteosarcoma cells. Oncotarget 6: 4920-4935, 2015.

19. Zhang H, Yin Z, Ning K, Wang L, Guo R and Ji Z: Prognostic value of microRNA-223/epithelial cell transforming sequence 2 signaling in patients with osteosarcoma. Hum Pathol 45: $1430-1436,2014$.

20. Huang YZ, Zhang J, Shao HY, Chen JP and Zhao HY: MicroRNA-191 promotes osteosarcoma cells proliferation by targeting checkpoint kinase 2. Tumour Biol 36: 6095-6101, 2015.

21. Fujiwara T, Katsuda T, Hagiwara K, Kosaka N, Yoshioka Y, Takahashi RU, Takeshita F, Kubota D, Kondo T, Ichikawa $\mathrm{H}$, et al: Clinical relevance and therapeutic significance of microRNA-133a expression profiles and functions in malignant osteosarcoma-initiating cells. Stem Cells 32: 959-973, 2014

22. Song QC, Shi ZB, Zhang YT, Ji L, Wang KZ, Duan DP and Dang XQ: Downregulation of microRNA-26a is associated with metastatic potential and the poor prognosis of osteosarcoma patients. Oncol Rep 31: 1263-1270, 2014.

23. Kobayashi E, Satow R, Ono M, Masuda M, Honda K, Sakuma T, Kawai A, Morioka H, Toyama Y and Yamada T: Microrna expression and functional profiles of osteosarcoma. Oncology 86: 94-103, 2014

24. Maire G, Martin JW, Yoshimoto M, Chilton-MacNeill S, Zielenska $M$ and Squire JA: Analysis of miRNA-gene expression-genomic profiles reveals complex mechanisms of microRNA deregulation in osteosarcoma. Cancer Genet 204: 138-146, 2011.

25. Kuijjer ML, Rydbeck H, Kresse SH, Buddingh EP, Lid AB, Roelofs $H$, Bürger $H$, Myklebost $O$, Hogendoorn $P C$, Meza-Zepeda LA and Cleton-Jansen AM: Identification of osteosarcoma driver genes by integrative analysis of copy number and gene expression data. Genes Chromosomes Cancer 51: 696-706, 2012.

26. Camps C, Saini HK, Mole DR, Choudhry H, Reczko M, Guerra-Assunção JA, Tian YM, Buffa FM, Harris AL, Hatzigeorgiou AG, et al: Integrated analysis of microRNA and mRNA expression and association with HIF binding reveals the complexity of microRNA expression regulation under hypoxia. Mol Cancer 13: 28, 2014.

27. Lu X, Dai C, Hou A, Cui J, Cheng D and Xu D: Dysregulated microRNA Profile in HeLa Cell Lines Induced by Lupeol. In: Bioinformatics Research and Applications. Springer, pp71-80, 2014.

28. Frigerio CS,Lau P, Salta E, Tournoy J, Bossers K, Vandenberghe R, Wallin A, Bjerke M,Zetterberg H,Blennow K and De Strooper B: Reduced expression of hsa-miR-27a-3p in CSF of patients with Alzheimer disease. Neurology 81: 2103-2106, 2013.

29. Chuang MK, Chiu YC, Chou WC, Hou HA, Chuang EY and Tien HF: A simple, powerful, and widely applicable Micro-RNA scoring system in prognostication of de novo myeloid leukemia patients. Blood 124: 71, 2014.

30. Chuang MK, Chiu YC, Chou WC, Hou HA, Chuang EY and Tien HF: A 3-microRNA scoring system for prognostication in de novo acute myeloid leukemia patients. Leukemia 29: 1051-1059, 2015.

31. Wang L, Zhu MJ, Ren AM, Wu HF, Han WM, Tan RY and Tu RQ: A ten-microRNA signature identified from a genome-wide microRNA expression profiling in human epithelial ovarian cancer. PLoS One 9: e96472, 2014

32. Hirata H, Ueno K, Shahryari V, Deng G, Tanaka Y, Tabatabai ZL, Hinoda $Y$ and Dahiya R: MicroRNA-182-5p promotes cell invasion and proliferation by down regulating FOXF2, RECK and MTSS1 genes in human prostate cancer. PLoS One 8: e55502, 2013.

33. Hirata H, Ueno K, Shahryari V, Tanaka Y, Tabatabai ZL, Hinoda Y and Dahiya R: Oncogenic miRNA-182-5p targets Smad4 and RECK in human bladder cancer. PLoS One 7: e51056, 2012.

34. Zhang K, Chu K, Wu X, Gao H, Wang J, Yuan YC, Loera S, Ho K, Wang Y, Chow W, et al: Amplification of FRS2 and activation of FGFR/FRS2 signaling pathway in high-grade liposarcoma. Cancer Res 73: 1298-1307, 2013.

35. Tania V, Ajay J, Naveen K, Steve D, Susan M and Gnanapragasam VJ: Role and expression of FRS2 and FRS3 in prostate cancer. BMC Cancer 11: 484, 2011. 
36. Luo LY, Kim E, Cheung HW, Weir BA, Dunn GP, Shen RR and Hahn WC: The tyrosine kinase adaptor protein FRS2 is oncogenic and amplified in high-grade serous ovarian cancer. Mol Cancer Res 13: 502-509, 2015.

37. Valencia T, Joseph A, Kachroo N, Darby S, Meakin S and Gnanapragasam VJ: Role and expression of FRS2 and FRS3 in prostate cancer. BMC Cancer 11: 484, 2011.

38. Wang J, Tsouko E, Jonsson P, Bergh J, Hartman J, Aydogdu E and Williams C: Abstract P4-07-12: miR-206 inhibits cell migration through direct targeting of the actin-binding protein coronin $1 \mathrm{C}$ in triple-negative breast cancer. Cancer Res 73 (24 Suppl): P4-07-12, 2013.

39. Mataki H, Enokida H, Chiyomaru T, Mizuno K, Matsushita R, Goto Y, Nishikawa R, Higashimoto I, Samukawa T, Nakagawa M, et al: Downregulation of the microRNA-1/133a cluster enhances cancer cell migration and invasion in lung-squamous cell carcinoma via regulation of Coronin1C. J Hum Genet 60: 53-61, 2015.

40. Novello C, Pazzaglia L, Cingolani C, Conti A, Quattrini I, Manara MC, Tognon M, Picci P and Benassi MS: miRNA expression profile in human osteosarcoma: Role of miR-1 and miR-133b in proliferation and cell cycle control. Int J Oncol 42: 667-675, 2013

41. Zhang C, Yao C, Li H, Wang G and He X: Serum levels of microRNA-133b and microRNA-206 expression predict prognosis in patients with osteosarcoma. Int J Clin Exp Pathol 7: 4194-4203, 2014.

42. Bao YP, Yi Y, Peng LL, Fang J, Liu KB, Li WZ and Luo HS: Roles of microRNA-206 in osteosarcoma pathogenesis and progression. Asian Pac J Cancer Prev 14: 3751-3755, 2013.
43. van Boxtel R, Gomez-Puerto C, Mokry M, Eijkelenboom A, van der Vos KE, Nieuwenhuis EE, Burgering BM, Lam EW and Coffer PJ: FOXP1 acts through a negative feedback loop to suppress FOXO-induced apoptosis. Cell Death Differ 20: 1219-1229, 2013.

44. Santo EE, Ebus ME, Koster J, Schulte JH, Lakeman A, van Sluis P, Vermeulen J, Gisselsson D, Øra I, Lindner S, et al: Oncogenic activation of FOXR1 by 11q23 intrachromosomal deletion-fusions in neuroblastoma. Oncogene 31: 1571-1581, 2012.

45. $\mathrm{Xu} \mathrm{H}$ and Liu B: CPEB4 is a candidate biomarker for defining metastatic cancers and directing personalized therapies. Med Hypotheses 81: 875-877, 2013.

46. Ortiz-Zapater E, Pineda D, Martínez-Bosch N, Fernández-Miranda G, Iglesias M, Alameda F, Moreno M, Eliscovich C, Eyras E, Real FX, et al: Key contribution of CPEB4-mediated translational control to cancer progression. Nat Med 18: 83-90, 2011 